\title{
ACCURATE METHOD OF MEASURING TRANSMITTED WAVE FREQUENCIES AT 5,000 AND 20,000 KILOCYCLES PER SECOND
}

\author{
By E. L. Hall
}

ABSTRACT

The measurement of the frequency of a radio transmitting station offers a convenient means of intercomparison of frequency standards. While there have been several articles published dealing with station frequency measurements, these have, for the most part, considered frequencies in the broadcast band or lower. This paper describes a method of measuring station frequencies applicable to any frequency, but dealing primarily with frequencies of approximately 5,000 and 20,000 kc. A high order of precision is obtained by the use of harmonics and audio-frequency beat notes. Most of the units of the equipment employed have been previously described in connection with other applications.

The measurement of the frequency of a radio transmitting station offers a means of intercomparison of the frequency standards of two or more laboratories, which may be in different countries, and of calibrating secondary frequency standards in various laboratories. The above applications have been used with increasing accuracy as improvements in radio apparatus and technique of measurement have become available. However, the accuracy obtainable in the intercomparison of two frequency standards in this manner may be surprisingly good if suitable measurement methods are chosen, as will be shown later.

In 1923 and 1924 simultaneous measurements were made upon the transmissions of some of the transoceanic telegraph stations by two European laboratories and the National Bureau of Standards. The agreement was of the order of 0.2 per cent.

The method of measurement described by Royden, ${ }^{1}$ using a frequency meter, is stated to readily give an accuracy of 0.1 per cent. While the frequency range covered is not stated, measurements at 14.5 and $20 \mathrm{kc}$ are mentioned.

A year ago the frequencies of the European broadcasting stations were being measured with an accuracy of 0.02 per cent, ${ }^{2}$ using the apparatus and methods described in papers by Braillard and Divoire ${ }^{3,4,5}$ Refinements in the method not usually used were said to increase the accuracy to a few thousandths of 1 per cent.

Frequency measurements upon broadcasting stations as described by Bogardus and Manning ${ }^{6}$ were made with an accuracy of about

1 George T. Royden, The Frequency Checking Station at Mare Island, Proc. I. R. E., 15, pp. 313-318;

W. H. F. Grimths, Accurate Wavemeter Design, Wireless World (London), 26, p. 113; January 29, 1930. - Braillard and Divoire, The Exact illand Precise Measurement of Wave Length in Radio Transmitting stations, Exp. Wireless \& Wireless Engineer (London), 4, pp. 322-330, 394-401; 1927.

4 R. Braillard and E. Divoire, How Broadcasting Wave Lengths are Checked. A Description of the international Listening Station at Brussels, Wireless World, 22, pp. 219-222; 1928.

${ }^{\circ} \mathrm{R}$. Braillard and E. Divoire, Measurement of Wave Lengths of Broadcasting Stations, Exp. Wireless Wireless Engineer (London), 6, pp. 412-421; 1929.

H. L. Bogardus and C. T. Manning, The Routine Measurement of the Operating Frequencies of 3roadcast Stations, Proc. I. R. E., 17, pp. 1225-1239; July, 1929. 
a hundredth of a per cent. The same order of accuracy is obtained by the method used in Italy by Pession and Gorio. ${ }^{7}$

Radio transmitting sets of a few years ago had no means of accurately controlling the frequency such as is now possible with a piezo control which is maintained at a constant temperature. If a radio transmitting set can be relied upon to maintain its frequency quite accurately, it is no longer necessary that simultaneous frequency measurements be made in order to obtain accurate frequency comparisons. There are probably very few technicians accustomed to making frequency measurements of high precision, who would guarantee to make such measurements at definitely prescribed times, because of various kinds of interference which invariably occur at such times. This is true at least in a radio laboratory where several may be engaged on various kinds of testing work at the same time, and electric motors and other apparatus may be producing interference. However, if the transmitting set remains constant, measurements can be made as convenient and consistent results can be obtained.

Some months ago the bureau undertook to measure the frequency of the transmitted wave of NKF, Bellevue, D. C., which was approximately $20,085 \mathrm{kc}$. This transmitting set is piezo-controlled by a quartz plate with a frequency of approximately $2,510 \mathrm{kc}$. Successive frequency doubling stages are employed. During preliminary measurements an antenna was temporarily connected in to the 5,000 ke stage and then to the $10,000 \mathrm{kc}$ stage, as well as to the final stage. The transmission could not be picked upon on the $10,000 \mathrm{kc}$ transmission, presumably because the bureau was within the skip-distance for this frequency and the ground wave was not of sufficient strength. As both the 5,000 and 20,000 kc transmissions could readily be picked up, it was thought desirable to have both these frequencies transmitted and use the measurements on one frequency as a check on the measurements at the other frequency. This was accomplished through the courtesy of Dr. Taylor and his associates at the Naval Research Laboratory.

The measurements here considered have been made upon special transmitted signals, the part used in measurement consisting of a long dash. While the method can be employed upon the keyed signal of a station, yet it loses its great precision in such a case, because it is difficult, if not impossible, to match a steady note precisely with an intermittent or broken note. Experience has shown the necessity of an identifying signal, such as the station's call letters, but these should be kept to a minimum, and the dashes for measurement purposes made to predominate.

Most of the apparatus used in the measurements has been described in other papers. ${ }^{8,9,10}$ The paper ${ }^{9}$ on testing piezo oscillators, states that the method described is also applicable to the measure-

7 G. Pession and T. Gorio, Measurement of the Frequencies of Distant Radio Transmitting Stations, Proc. I. IR. E., 17, pp. 734-744; A pril, 1929, and Sulla Misura Della Lunghezza D'Onda, Pession and Gorio, Hilettrotecnica (Italy), 15, pp. 524-530; 1928.

8 E. L. Hall, A System for Frequency Measurements Based on a Single Frequency, Proc. I. R. E., 17, pp. 272-282; February, 1929.

E. L. Mall, Method and Apparatus Used in Testing Piezo Oscillators for Broadcasting Stations, B. S. Jour. Research, 4, pp. 115-130; January, 1930; also as Research Paper No. 135, obtainable from Superintendent of Documents, Government Printing Office, Washington, D. C., for 10 cents. Proc. I. R. E., 1s, pp. 490-509; Narch, 1930.

10 N. P. Case, A Precise and Rapid Method for Measuring Low Frequencies, B. S. Jour. Research, 5, (RP195), p. 237, 1930; and the Proc. I. R. E. 
ment of station frequencies. Since the paper was prepared, the bureau has obtained a very accurate primary standard of frequency, similar to that described by Marrison. ${ }^{11}$ The frequency measurements are based upon this new standard rather than upon the standard mentioned in the previous reference. ${ }^{9}$

'The method of measurement is the familiar one consisting of tuning a receiving set to the frequency of the station to be measured, adjusting a radio-frequency generator to the same frequency or some harmonic of it, and measuring the frequency of the generator. The frequency relation between the generator and the receiving set being known, the frequency of the signal is readily obtained.

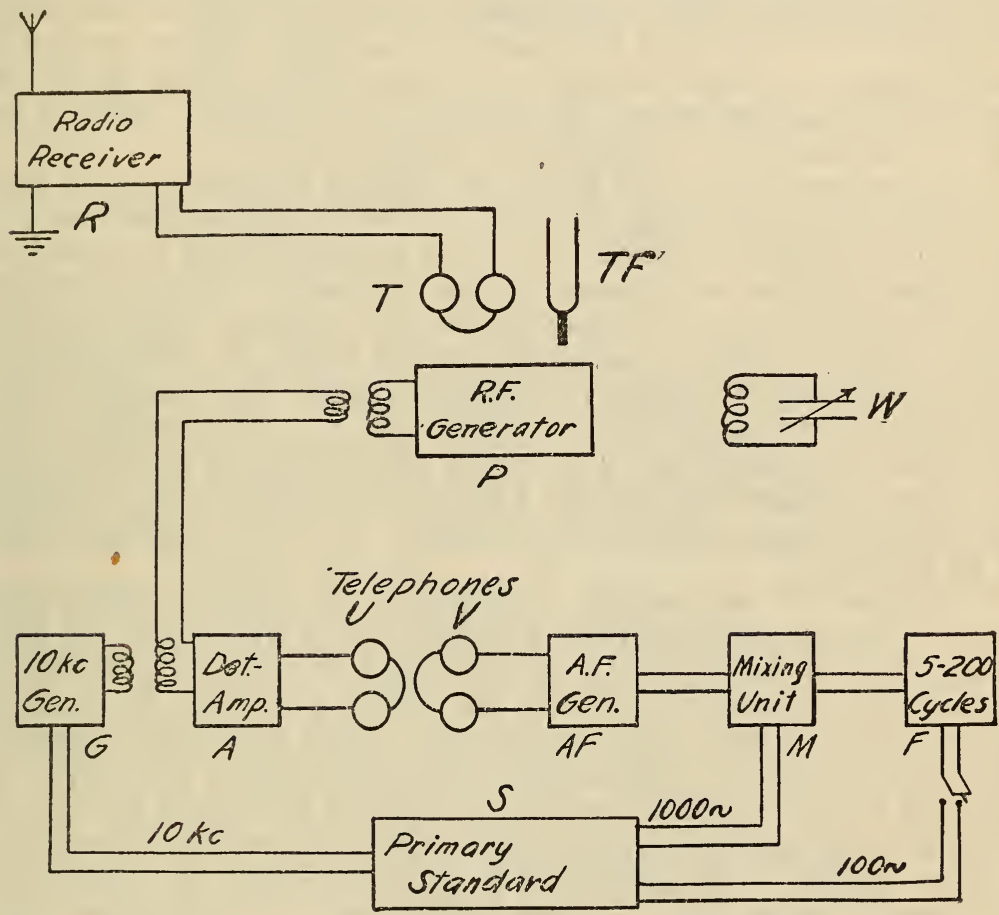

FIgURE 1.-Schematic diagram of apparatus used in measurement of station frequencies in terms of the primary frequency standard

In order to explain the refinements making for increased precision and accuracy in the measurements to be described, reference is made to Figure 1. The radio receiver $R$ is adjusted to the desired station signal, as for example, $5021 \mathrm{kc}$, and left in a nonoscillating condition. The generator $P$ is then adjusted to "zero beat" with the radio receiver, and a reading taken upon the frequency meter $W$. Generator $P$ is then adjusted away from "zero beat" until beats are obtained by matching with the note from a tuning fork $T F$, which is held in the hand and struck with a small mallet as desired. A second reading is taken at this time upon the frequency meter $W$, the readings of which are used in recording the direction from zero

See footnote 9 , p. 648 .

11 W. A. Marison, A High Precision Standard of Frequency, Bell System Tech. J., 8, pp. 493-514: July, 1929; also Proc. I. R. E., 17, pp. 1103-1122; July, 1929. 
beat where the precise measurement is made. Further discussion of the frequency at which generator $P$ is set for the measurement will be given after describing the remainder of the procedure.

The $10 \mathrm{kc}$ generator $G$ is controlled by the primary standard $S$ which supplies current at $10 \mathrm{kc}$. Generator $G$ is of the Hartley type, having a grid resistor across which the control voltage is impressed. It remains in control over a considerable capacity change. Harmonics of the $10 \mathrm{kc}$ generator $G$ have been used in measurements as high as the 290th, but, generally speaking, harmonics below half this order are usually used.

The detector-amplifier, described in a previous paper, ${ }^{12}$ picks up the beat frequency between generator $P$ and generator $G$, as heard in telephones $U$. This beat frequency is matched with a similar frequency from the audio-frequency generator $A F$ as heard in the telephones $V$, at a time when the setting of generator $P$ gives a beat note in the telephones $T$ matching tuning fork $T F$.

The characteristics of the generator $A F$ are such that it will maintain its frequency over long periods. However, the calibration is not relied upon, and the output is sent into a mixing unit $M$ or modulator, which also receives a thousand cycles per second output from the primary standard $S$. The resulting beat frequency output from $M$ is sent into the direct-reading frequency meter $F$, described in a previous reference, ${ }^{13}$ which is checked against the hundred cycles per second output of the primary standard $S$. The frequency meter $F$ is limited in range from 5 to about 200 cycles per second, but by use of the modulator $M$ and the 1,000-cycle output from $S$, measurements in this range around the harmonics of 1,000 cycles per second can be made.

If it is desired to measure a frequency of approximately 20,085 $k c$, for example, with generator $P$ set at such a frequency, the detector-amplifier $A$ could hardly be expected to pick up the 2,008th harmonic of generator $G$. If it did, there would be an audio-frequency note of 5,000 cycles per second, which can not be measured with this equipment. Harmonics of the generator $P$, however, will be picked up by the radio receiver $R$, so that if $P$ is set at $1 / 40$ of $R$ or $502 \mathrm{kc}$, the beat notes work very nicely throughout the system. In other words, when generator $P$ is off of $1 / 40$ the frequency of $R$ to match tuning fork $T F$, the telephones $U$ connected to $A$ produce a note against a harmonic of the standard 10 kc generator $G$, which can readily be matched with the note in telephones $V$ and checked up with the frequency meter $F$, coming within the range of $2,000 \pm 200$ cycles per second. Tuning forks of several frequencies have been used to obtain check values agreeing to the order of 1 . part in $10^{6}$.

For check measurements at $5,021 \mathrm{kc}$, generator $P$ is set in $\Omega$ similar manner. If the same tuning fork is used, a slightly different beat frequency is produced from that at $20,085 \mathrm{kc}$. In order to make measurements with the above apparatus at a given frequency, the harmonics and beat notes employed must bear certain relations to each other, as are stated below. 
Let-

$X=$ frequency to be measured, which is approximately known from frequency assignment.

$H=$ frequency to which generator $P$ is adjusted.

$A=$ audio-frequency note produced after generator $P$ is precisely

adjusted in certain relationship to frequency $X$.

$A$ should have one of the values previously mentioned; that is,

5 to $200,1,000 \pm 200,2,000 \pm 200$, etc., cycles per second.

$H$ must have a value such that-

(1) $A$ is satisfied.

(2) Beat note between generator $P$ and $10 \mathrm{kc}$ generator $G$ comes in the range preferably from 100 to 2,500 cycles per second.

(3) Beat note between harmonic of $H$ and frequency $X$ should come between 400 and 1,500 cycles per second. in order to be easily matched.

(4) The frequency setting to be preferably under 1,000 kc. (Measurements have been made at three times this frequency.)

While the above conditions may seem to be quite formidable, in practice several solutions may be possible. Several measurements are possible by varying (3), using tuning forks of different frequencies.

Data taken on the piezo-controlled transmitting set at NKF upon frequencies of approximately $5,021 \mathrm{kc}$ and $20,085 \mathrm{kc}$ gave practically the same results by the above method, although five different conditions as to beat note produced were used. It was of interest to find that measurements made at the Naval Research Laboratory by an entirely different method and in terms of a different standard, agreed with our results within about 2 parts in $10^{6}$.

A consideration of the possibilities of error in the measurements may be of interest. Consider the measurement of 20,085.000 kc. The radio receiver, when properly set, will not introduce an error. If the radio-frequency generator was set at $1 / 40$ the frequency to which the radio receiver was adjusted, it would be set at $502.125 \mathrm{kc}$. As it can not be set accurately here, the 40th harmonic of the generator is made to produce a 1,000 cycle difference frequency with the incoming signal. If the generator is set low, its frequency will be $502.125-\frac{1,000}{40}=502.100 \mathrm{kc}$. If the 1,000 -cycle beat note is held to 1 cycle per second, the generator is set to $1 / 40$ cycle per second, or to $502.100 \mathrm{kc} \pm 0.025$ cycles. It is quite difficult, if not impossible, to hold the generator to such precision. If the generator is held so that there is a beat of 1 cycle per second, which is not impossible, the error in setting will be 1 part in $2 \times 10^{7}$. The error in matching the note between the standard and the generator, with the audiofrequency generator, is of considerable importance, because instead of affecting the result by $1 / 40$, it affects it directly. The error in this matching is multiplied by 40 with the frequencies considered. However, it is possible to make the matching quite accurately, providing the radio-frequency generator holds, or the matching is carefully made when the 1,000-cycle beat note is correct. Changing the setting of a variable air condenser either side of zero beat and taking the mean setting gives a precise adjustment. The setting 
of the radio-frequency generator affects the ease of this matching by a factor of 50 in this case, because the note which is matched with the audio-frequency generator is produced by the fundamental frequency $(500 \mathrm{kc})$ of the generator beating with the 50 th harmonic of the $10 \mathrm{kc}$ generator. A change of the generator frequency of $1 / 40$ cycle, which represents a change of 1 part in $2 \times 10^{7}$ with respect to the measured frequency, here produces a change of approximately 1 part in $10^{5}$. If referred to the measured frequency this would represent a change of $0.2 \mathrm{kc}$, which is about five times the difference obtained in measured values for a given day. The factor 50 , therefore, does not enter into the calculated error so much, but has more to do with the difficulties of making the measurement.

The audio-frequency generator output is then determined in terms of the direct-reading frequency meter. Errors which may be inherent in this device are likewise multiplied by 40 in arriving at the final result.

In the absence of more data the following estimates of errors in measurement may be listed. The total value thus obtained is of the same order as the departure from the mean values of frequency

Estimated error in adjusting generator to receiver

Parts in $10^{7}$

Estimated error in adjusting audio-frequency generator to generator \pm 0.5 Estimated error in measuring audio-generator note.

Total

As the sign of the errors may be either positive or negative, some of them tend to cancel others.

The high precision and accuracy of the measurements of station frequencies by the above method arises from the use of harmonics and beat notes plus an acquired skill in the precision of setting the various parts of the equipment. The use of several tuning forks in making the settings verifies the results obtained, and in spite of a large multiplying factor, as previously stated, which applies to the errors present, the results usually agree to about 2 parts in $10^{6}$.

Measurements on lower frequencies, such as in the broadcasting band, can be made very precisely in the above manner, and have the advantage of a small multiplying factor.

Washington, March 25, 1930. 